ARTICLE

Received 4 Feb 2015 | Accepted 7 Aug 2015 | Published 15 Sep $2015 \quad$ DOl: 10.1038/ncomms9286 OPEN

\title{
Efficient direct solar-to-hydrogen conversion by in situ interface transformation of a tandem structure
}

Matthias M. May ${ }^{1,2,3}$, Hans-Joachim Lewerenz ${ }^{4}$, David Lackner ${ }^{5}$, Frank Dimroth ${ }^{5} \&$ Thomas Hannappel $^{2}$

Photosynthesis is nature's route to convert intermittent solar irradiation into storable energy, while its use for an industrial energy supply is impaired by low efficiency. Artificial photosynthesis provides a promising alternative for efficient robust carbon-neutral renewable energy generation. The approach of direct hydrogen generation by photoelectrochemical water splitting utilizes customized tandem absorber structures to mimic the Z-scheme of natural photosynthesis. Here a combined chemical surface transformation of a tandem structure and catalyst deposition at ambient temperature yields photocurrents approaching the theoretical limit of the absorber and results in a solar-to-hydrogen efficiency of $14 \%$. The potentiostatically assisted photoelectrode efficiency is $17 \%$. Present benchmarks for integrated systems are clearly exceeded. Details of the in situ interface transformation, the electronic improvement and chemical passivation are presented. The surface functionalization procedure is widely applicable and can be precisely controlled, allowing further developments of high-efficiency robust hydrogen generators.

\footnotetext{
${ }^{1}$ Helmholtz-Zentrum Berlin für Materialien und Energie GmbH, Institute for Solar Fuels, Hahn-Meitner-Platz 1, D-14109 Berlin, Germany. ${ }^{2}$ Department of Physcis, Technische Universität IImenau, Gustav-Kirchhoff-Str. 5, D-98693 IImenau, Germany. ${ }^{3}$ Department of Physcis, Humboldt-Universität zu Berlin, Newtonstraße 15, D-12489 Berlin, Germany. ${ }^{4}$ California Institute of Technology, Joint Center for Artificial Photosynthesis, 1200 East California Boulevard, Pasadena, California 91125, USA. ${ }^{5}$ Fraunhofer Institute for Solar Energy Systems ISE, Heidenhofstraße 2, D-79110 Freiburg, Germany. Correspondence and requests for materials should be addressed to H.J.L. (email: lewerenz@caltech.edu) or to T.H. (email: thomas.hannappel@tu-ilmenau.de).
} 
H ydrogen provides the highest energy density among the common fuels ${ }^{1}$. In the search for sustainable, low-carbon replacements for fossil fuels, solar water splitting is receiving exceptional attention, with $\mathrm{H}_{2}$ as the crucial ingredient of an anthropogenic carbon cycle or a completely carbon-free energy economy ${ }^{1,2}$. Solar energy is stored in chemical bonds by splitting $\mathrm{H}_{2} \mathrm{O}$ photoelectrochemically into $\mathrm{H}_{2}$ and $\mathrm{O}_{2}$. The associated thermodynamic potential difference of $1.23 \mathrm{~V}$ must be exceeded by the free energy of the charge carriers transferred to the electrolyte ${ }^{3}$. The required, minimum photovoltage for the photolysis of water is $\gtrsim 1.6 \mathrm{~V}$, including catalyst overpotentials, and depends on the chosen photocurrent density 4 . Since excess energy is dissipated as heat, the chemical energy stored in a single $\mathrm{H}_{2}$ molecule is $2 \times 1.23 \mathrm{eV}$. Overcoming the voltage threshold and utilizing a constant energy per reduced hydronium ion requires photocurrent maximization, if the photovoltage suffices to drive the reaction. These boundary conditions make tandem photovoltaic devices, where semiconductor absorber layers with different energy gaps are combined for enhanced exploitation of the solar spectrum, superior candidates for photoelectrochemical water splitting. As a highly efficient, inorganic analogue to the Z-scheme of natural photosynthesis ${ }^{5}$, dual-tandem structures exhibit increased photovoltages while simultaneously exploiting the sunlight efficiently, thus permitting high photocurrents. In contrast, photovoltaic power generation is less restricted as the utilizable output power is defined via the current-voltage product. Stacking multiple absorbers can increase both photovoltage and delivered power at the expense of the achievable current ${ }^{6-8}$. Despite employing relatively high-cost, but high-efficiency structures, solar $\mathrm{H}_{2}$ is predicted to become competitive at solar-to-hydrogen (STH) efficiencies of $15 \%$ and beyond ${ }^{9}$.

Two principle approaches for solar water splitting have been advocated: the non-monolithic approach, where photovoltaic light harvesting and electrolytic water splitting are spatially separated, or monolithically integrated devices, which are fully immersed into the electrolytes. The former one avoids issues associated with the semiconductor-electrolyte contact, such as the challenge to employ heterogeneous catalysts with low light absorption $^{10}$ and photocorrosion, but necessitates a second technology line ${ }^{11}$. Whereas with the former approach, STH efficiencies of up to $18 \%$ have been achieved ${ }^{12,13}$, the benchmark for monolithic water splitting is at $12.4 \% \mathrm{STH}$ for 11 suns illumination, and achieving simultaneously efficiency and stability remains an issue ${ }^{14}$.

For the integrated, direct approach pursued here, the photolysis cell design is more demanding, but is alleviated by a higher potential for cost reduction of solar $\mathrm{H}_{2}$ generation ${ }^{9,15}$. An extensive overview of existing systems and their performance can be found in the literature, though sometimes no clear distinction between monolithic and non-monolithic systems is made there ${ }^{16}$.

Selection of the tandem absorber energy gaps for an efficient use of the solar spectrum and simultaneous supply of the necessary photovoltage for water photolysis yields optimum energy gap combinations in the range of $0.8-1.2$ and $1.5-1.9 \mathrm{eV}$ for the bottom and top cells, respectively ${ }^{7,17}$. For III-V semiconductors, their adjustable optoelectronic properties, the high control of doping levels, the formation of tunnel junctions and abrupt interfaces enable such an adaptation ${ }^{18-20}$. Application in photoelectrochemical devices necessitates in addition a careful conditioning of the interfaces of the absorber with the electrolyte and with the electrocatalyst, also considering molecular details of the surface chemistry ${ }^{21,22}$. The interface modification has to simultaneously provide corrosion protection, high optoelectronic quality, sufficient optical transparency as well as a suitable mechanical and electronic coupling to the catalyst ${ }^{7,23,24}$. In situ (photo)electrochemical functionalization is a low temperature, ambient pressure wet processing method. Similar to galvanic processing, it is widely applicable and, in principle, industrially scalable. It has already been shown that wet semiconductor processing can provide high-quality interfaces combined with high stability 7,23 .

We present here an in situ surface functionalization routine developed for a III-V photovoltaic tandem absorber, that enables an STH effiency of $14 \%$ for unbiased, direct solar water splitting. Electronic and chemical passivation are achieved via a transformation of the surface AlInP layer towards oxides and phosphates/phosphites, which allow efficient coupling with the $\mathrm{Rh} \mathrm{H}_{2}$ evolution electrocatalyst. A further reduction of interfacial charge-carrier recombination would unlock the full potential of the device reaching efficiencies beyond $17 \%$, which is predicted to become interesting for commercial prototypes ${ }^{9}$.

\section{Results}

Customized tandem absorber. A two-junction tandem absorber structure, grown epitaxially on a Ge substrate by metal-organic vapour phase epitaxy ${ }^{19}$, serves as photovoltaic core element. The absorber consists of a GaInP $n-p$ top cell with an energy gap of $E_{g}=1.78 \mathrm{eV}$ and of an $n-i-p$ GaInAs bottom cell with $E_{g}=1.26 \mathrm{eV}$ (Fig. 1a). The structure also contains cap layers and an AlInP window layer for electron collection. In the photovoltaic mode and with anti-reflection coating, a shortcircuit current density of up to $15 \mathrm{~mA} \mathrm{~cm}^{-2}$ and an open-circuit (OC) voltage of $2.1 \mathrm{~V}$ is expected at AM $1.5 \mathrm{G}$ illumination ${ }^{19}$. The energy band relations are depicted in Fig. 2. They show the energetic situation near the maximum power point of the device where, for high fill factors, the photovoltage is close to the OC voltage, but the photocurrent is still near its maximum (short circuit) value. The photovoltage generated is represented by the a

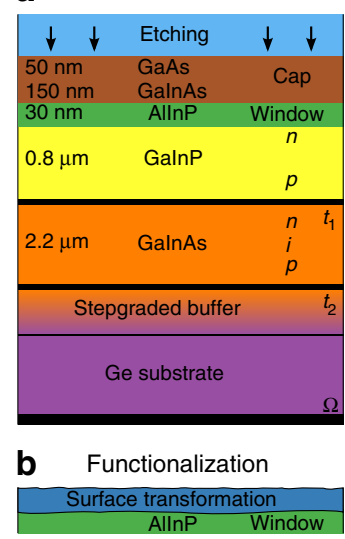

C

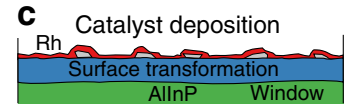

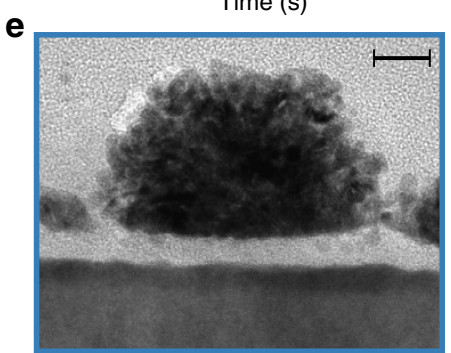

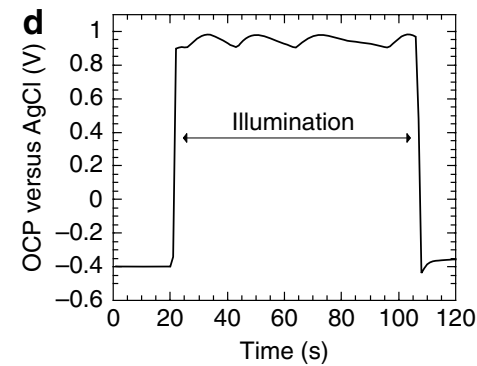

Figure 1 | Interfacial functionalization steps. The tandem consists of a GalnP $n-p$ top cell and a GalnAs $n-i-p$ bottom cell. (a) Stacking and thicknesses; $t_{1}$ and $t_{2}$, and $\Omega$ denote tunnel junctions and ohmic back contact, respectively; vertical arrows indicate selective chemical etching of the capping layer. (b) Chemical and photoelectrochemical surface transformation of the AllnP window layer in an aqueous solution of $\mathrm{RhCl}$. (c) Electrochemical deposition of a seed layer of Rh electrocatalysts (grey) and photoelectrodeposition of a continuous electrocatalyst film (red; see text). (d) Oscillation of the open-circuit potential on illumination during functionalization. (e) Transmission electron microscope image of a cross-section of the surface after Rh catalyst deposition; scale bar, $10 \mathrm{~nm}$. 


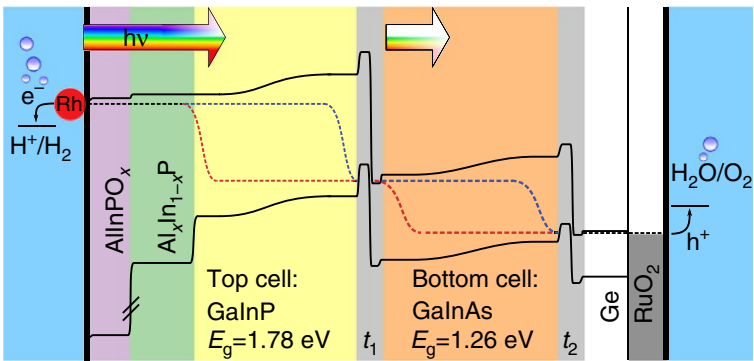

Figure 2 | Energy schematic of the tandem layer structure under illumination. The ohmic back contact is connected to a sputtered $\mathrm{RuO}_{2}$ counter electrode, where $\mathrm{O}_{2}$ evolution occurs. $\mathrm{H}_{2}$ evolution takes place at the interface of the Rh electrocatalyst on top of the functionalized layer with the electrolyte. Ohmic contacts between subcells are made by tunnel junctions, where switching between majority-charge-carrier types occurs. Black, dashed lines represent the Fermi level, blue (red) the Quasi-Fermi levels of electrons (holes) and arrows indicate the impinging light.

difference of the quasi-Fermi levels of electrons (blue curve in Fig. 2) and holes (red curve), yielding the achievable free energy of the system near the maximum power point. The structure operates as photocathode with a dark anode using a $\mathrm{RuO}_{2}$ catalyst for $\mathrm{O}_{2}$ evolution.

Interface functionalization. The fundamental surface processing steps are summarized in Fig. 1a-c. A protective cap layer was removed by selective etching ${ }^{25}$ in a solution of $\mathrm{NH}_{4} \mathrm{OH}: \mathrm{H}_{2} \mathrm{O}_{2}: \mathrm{H}_{2} \mathrm{O}$, stopping at the $\mathrm{n}^{+}$-doped $\mathrm{Al}_{0.35} \mathrm{In}_{0.65} \mathrm{P}$ window layer. A suitable surface for the subsequent functionalization was prepared by chemical oxidation in $\mathrm{O}_{2}$-saturated water, followed by a drying process in a flow of oxygen, resulting in a smooth, oxidized surface. The surface composition was analysed by X-ray photoelectron spectroscopy (XPS), revealing a mixed In/Al oxide layer on top of the AlInP window (Fig. 3). Such oxide layers typically form a barrier for holes, reducing charge-carrier recombination ${ }^{7,23}$.

Combined chemical and photoelectrochemical surface conditioning is essential to increase stability and charge transfer efficiency, as the stability of a chemically formed, pure group III element ( $\mathrm{In}$ and $\mathrm{Al}$ ) oxide layer, which is the outcome of the etching process (Figs 1a and 3), can be rather limited under operating conditions ${ }^{26}$. We developed an advanced, but straightforward procedure that allows for simultaneous surface functionalization and catalyst deposition in a single solution in a two-step approach (Fig. 1b-d). As in situ process, the method also prevents unfavourable oxidation from ambient $\mathrm{O}_{2}$ (ref. 22). Under OC conditions, the sample was illuminated with white light, initiating an oscillation (Fig. 1d) of the observed potential. Oscillation periods were in the range of $20 \mathrm{~s}$ with amplitudes in the order of $100 \mathrm{mV}$. After typically 5-6 oscillations, the OC potential reached its maximum. We attribute this phenomenon to a slow layer-by-layer etching, where surfaces with superior electronic quality are simultaneously prepared. The process shows similarity to the current-oscillations observed at the $\mathrm{Si}$-electrolyte contact ${ }^{27,28}$ : in this model, the anodic and the cathodic currents equilibrate at OC; the anodic partial current leads to oxide formation in competition with the cathodic processes of $\mathrm{H}_{2}$ evolution, excess minority carrier recombination, and the reduction of formed oxides. Oscillations occur if, within one cycle, the locally formed initial oxide islands are reduced more slowly, hence are more stable than the later formed ones, which exhibit more strain-induced defects. This constitutes a feedback mechanism that synchronises the oxidation and the cathodic etching process. This hypothesis is supported by the
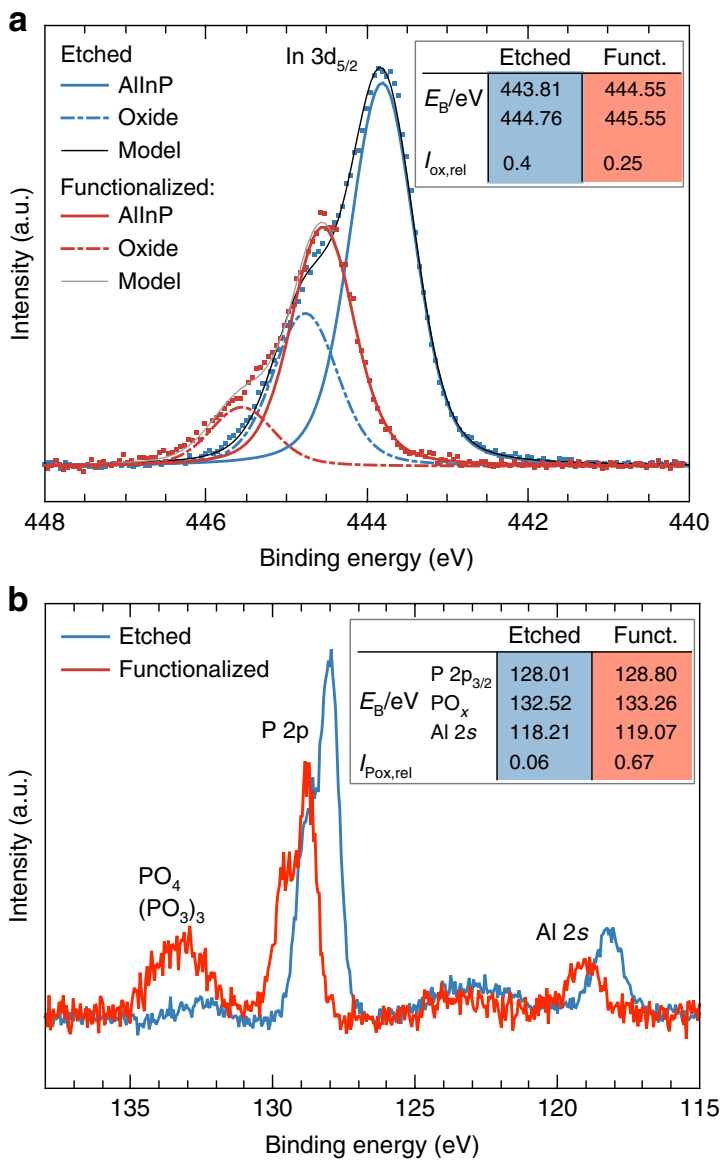

Figure 3 | Surface analysis after functionalization by XPS. (a) Spectrum of the $\ln 3 d_{5 / 2}$ core level after chemical etching of the capping layer, before (blue curve) and after (red curve) complete functionalization revealing a decrease of In content and composition (see text). (b) P 2p and Al $2 s$ core levels unveiling a transformation of $\mathrm{P}$ in AllnP towards phosphite/ phosphate species.

finding that at the OCP maximum, the thickness of the oxide is reduced to $0.4 \mathrm{~nm}$, in comparison with $0.8 \mathrm{~nm}$ at the OCP minimum (Supplementary Fig. 1). Analogous formation of intermediate islands, mediated by surface defects, has also been observed for the removal of oxides from III-V surfaces in gasphase experiments ${ }^{29}$. The periodic change of the photovoltage is attributed to periodic thickness variations, corresponding potential drops across the interfacial film, and periodically increased recombination within a cycle when more defect-rich films are formed. The photovoltage is highest for the least oxidized surface.

The photovoltage oscillation step was directly followed by electrodeposition of the $\mathrm{Rh}$ electrocatalyst. The deposition was performed by applying potential pulses of $50 \mathrm{~ms}$ duration over a time of typically $30 \mathrm{~s}$ in the dark (see the Methods section). Subsequently, photoelectrodeposition of Rh was carried out by periodically varied illumination between low and high light intensity, followed by $30 \mathrm{~s}$ in the dark (Supplementary Fig. 2). The oscillating behaviour of the OCP on illumination during the functionalization is not a unique feature of the Rh-containing solution, but can also be induced in pure $\mathrm{HClO}_{4}$. From a device perspective, however, the beneficial effect of the oscillation on the surface is maximized if the catalyst deposition is conducted directly thereafter, that is, in the same electrolyte.

The process resulted in a composite structure of seed catalyst nanoparticles and a thin Rh covering layer (Fig. 1c,e). XPS 


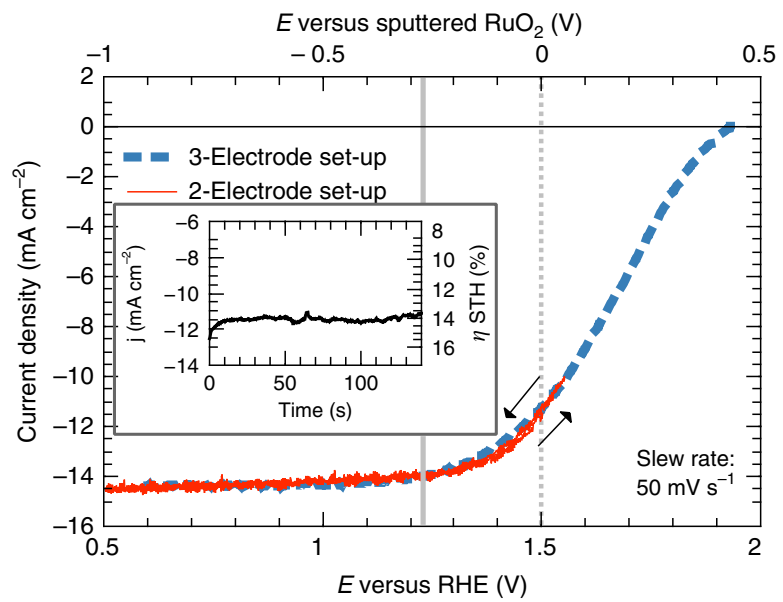

Figure 4 | Output power characteristic. The in situ modified two-junction tandem structures were evaluated in $1 \mathrm{M} \mathrm{HClO}_{4}$ under $\mathrm{AM} 1.5 \mathrm{G}$ illumination. The red curve shows a two-electrode configuration with a $\mathrm{RuO}_{2}$ counter electrode. The blue curve represents a cyclic voltammogram in a three-electrode set-up with Pt counter electrode plotted versus the reversible hydrogen electrode redox potential (RHE). The grey, solid line marks $+1.23 \mathrm{~V}$ versus $\mathrm{RHE}$ and the dashed line $\mathrm{OV}$ versus $\mathrm{RuO}_{2}$. The curves intersect at $\mathrm{OV}$ versus $\mathrm{RuO}_{2}$ and $1.5 \mathrm{~V}$ versus RHE. The inset depicts the current over time for the unbiased two-electrode configuration.

revealed that the initial surface functionalization before $\mathrm{Rh}$ deposition led to a reduction of the In content at the surface and, preferentially, of the relative In oxide contribution (Fig. 3a). While the $\mathrm{Al} 2 s$ line (Fig. $3 \mathrm{~b}$ ) is slightly reduced and develops a small shoulder towards higher binding energies, the P $2 p$ signal shows a partial transformation into a mixture of $\mathrm{InPO}_{4}$ and $\mathrm{In}\left(\mathrm{PO}_{3}\right)_{3}$, indicated by the peak between 133 and $134 \mathrm{eV}$ binding energy ${ }^{7,30}$. Peak analysis shows that the relative $\mathrm{PO}_{x}$ contribution is increased from initially 6 to $67 \%$ after functionalization.

Photoemission lines of the functionalised surfaces are, compared with those of the etched samples, shifted by $(0.75 \pm 0.1) \mathrm{eV}$ to higher binding energies (Fig. 3). This shift originates in part from the higher $\mathrm{n}$-doping of the functionalised, phosphate- and phosphite-rich surface, also observed for InP photocathodes ${ }^{7}$.

For the oxidized sample directly after etching, the Fermi level at the topmost surface is energetically lowered in comparison with the highly n-doped AlInP bulk due to a lower doping and a higher electron affinity of the oxide ${ }^{18,31}$. The completely functionalised surface that was transformed towards $\mathrm{PO}_{x}$, on the other hand, exhibits a higher $\mathrm{n}$-doping and the Fermi level is shifted towards the vacuum level (cf. Fig. 3 and Supplementary Fig. 3), improving the band alignment to the AlInP. The beneficial effect of the $\mathrm{PO}_{x}$ phase is further supported by the observation that the signal strength of this species (Fig. 3b) is directly correlated with the overall efficiency. In a quantitative analysis of the $\mathrm{P} 2 p$ lines, the average thickness of the phosphate and phosphite layer was estimated to be $(1.3 \pm 0.2) \mathrm{nm}$.

An analysis of transient photovoltages (Supplementary Fig. 4) shows an overshoot for not fully transformed surfaces, which indicates a trapping of charge carriers in surface states ${ }^{32}$. Together with the finding, that OC voltages increase significantly for devices with $\mathrm{PO}_{x}$ termination (cf. Supplementary Fig. 5), we conclude that the functionalization layer also provides an electronic surface passivation. This transformation is a feature of the in situ functionalization before catalyst deposition, which avoids the formation of charge-carrier recombination centres at strained In/Al-O-In/Al bonds, that would form upon exposure to

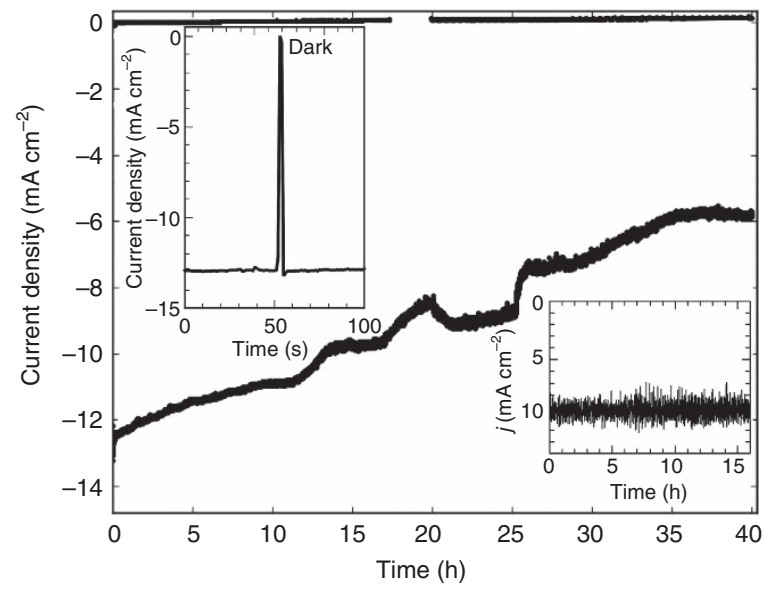

Figure 5 | Stability assessment. The AM 1.5G illumination in a vertical electrode geometry was chopped with $5 \mathrm{~s}$ dark time and $200 \mathrm{~s}$ exposure. Left inset: zoom into the first light-dark cycle. Right inset: current evolution in a horizontal, non-chopped three-electrode configuration. Potential $+600 \mathrm{mV}$ versus RHE.

atmospheric $\mathrm{O}_{2}$ (refs 24,22), and turned out to be a crucial prerequisite for high efficiency.

Cell performance. The output characteristics of the resulting devices (stacking and energy schematic sketched in Figs 1 and 2) were evaluated in $1 \mathrm{M} \mathrm{HClO}_{4}$ under simulated sunlight (AM $1.5 \mathrm{G}$, light intensity $I=100 \mathrm{~mW} \mathrm{~cm}^{-2}$; Fig. 4). Both, results of a two-electrode and a three-electrode potentiostatic measurement are shown. For the former, a current density of $j=11.5 \mathrm{~mA} \mathrm{~cm}^{-2}$ without bias is reached, as seen in the inset. With the definition of the solar-to-hydrogen efficiency, $\eta=\left(j_{\mathrm{H}_{2}} \times 1.23 \mathrm{~V}\right) / I$, this translates into an efficiency of $\eta=14 \%$ under the assumption of $100 \%$ Faradaic efficiency. A saturation current density beyond $14 \mathrm{~mA} \mathrm{~cm}^{-2}$, measured in a three-electrode set-up, is close to its maximum value, indicating that light transmission through the catalyst is not a major issue. The OC potential of $1.9 \mathrm{~V}$ is, however, $200 \mathrm{mV}$ below the expected value, which is mainly ascribed to interfacial charge-carrier recombination. In conjunction with the linear exponential catalyst characteristic, this results in the S-shaped onset of the photocurrent ${ }^{33}$. The difference between two- and three-electrode set-up measurements is attributed to the overpotential of the oxygen evolution reaction as well as the solution resistance. The observation of $14 \mathrm{~mA} \mathrm{~cm}^{-2}$ at $+1.23 \mathrm{~V}$ versus RHE results in an assisted photocathode efficiency of $>17 \%$ for the three-electrode arrangement (blue curve in Fig. 4), demonstrating the potential efficiency of the device. An example for an incomplete functionalization towards $\mathrm{PO}_{x}$ is given in the Supplementary Fig. 5, showing a significantly reduced fill factor. A successful surface transformation combined, however, with catalyst overloading results in reduced optical transmission and an associated reduction of the photocurrent, while the OC voltage is increased. A total of $8 \%$ of the samples, which had an active surface area between 30 and $100 \mathrm{~mm}^{2}$, showed highly successful functionalization with photocurrent densities of $11 \mathrm{~mA} \mathrm{~cm}^{-2}$ and beyond, $25 \%$ exceeded $10 \mathrm{~mA} \mathrm{~cm}^{-2}$ (see also the Methods section).

Chronoamperometric data over $40 \mathrm{~h}$ are displayed in Fig. 5. For a vertical configuration, the photocurrent density degrades continuously, saturating at 0.5 of its original value after $35 \mathrm{~h}$ of operation. Optical inspection of the electrode surface shows that steps in the decrease of the current are associated with severe detachment of catalysts from the surface, likely a result of 
chemical etching and stress from $\mathrm{H}_{2}$ bubbles propagating parallel to the surface, as already proposed in ref. 14. In a horizontal set-up (with slight catalyst overloading as indicated by only $j=10 \mathrm{~mA} \mathrm{~cm}^{-2}$ ), no degradation of the photocurrent for over $16 \mathrm{~h}$ of operation is noted (see inset of Fig. 4).

These data show the hitherto best stability reported for a monolithically integrated, high efficiency, fully immersed device. Longer stability, obtained with half cells $s^{23,34}$ cannot directly be compared with those of tandem structures because the absorbers used in those experiments were high-quality single-crystalline $\mathrm{Si}, \mathrm{GaAs}$, InP and GaP. In heteroepitaxy for tandem cells, multi-stack layer growth results in growth-induced defects that can protrude to the surface. Such sites can act as nucleation centres for subsequent defects, which develop during surface transformation, electrodeposition and finally operation. Resulting pinhole formation leads to a reduced mechanical stability of the $\mathrm{Rh}$ catalyst due to undercutting at sites, where the corrosion protection layer has been incompletely formed. The mechanical detachment of the catalyst is more severe in a vertical configuration of the electrode, where gas bubbles, which have been observed to move directly along the surface, induce optical and mechanical interaction in particular at defect sites and further local corrosion. Our results point to the necessity to prepare custom-designed tandem absorber structures because the difference in surface chemistry at defect sites will also limit protection possibilities via atomic layer deposition. A possible solution could be the deposition of thin layers of earth-abundant materials by physical vapour deposition followed by under potential deposition ${ }^{35}$ of the catalyst, which would also reduce the overall noble-metal consumption.

High Faradaic solar-to-hydrogen efficiency is confirmed by the measurement of gas evolution (Fig. 6a), exhibiting a 2:1 ratio of $\mathrm{H}_{2}$ to $\mathrm{O}_{2}$ and an equivalent of the evolved gases to the overall charge transferred within the measurement error of ca. $5 \%$.


Figure 6 | Gas evolution and surface morphology. (a) Gas evolution of the illuminated device under galvanostatic conditions. (b) Rh catalyst nanoparticles at the surface after the passage of $-40 \mathrm{Ccm}^{-2}$ under operation by scanning electron microscopy (backscattering image). Black areas show the underlying absorber and grey areas the catalyst nanoparticles; scale bar, $200 \mathrm{~nm}$.

\section{Discussion}

To estimate the Faradaic efficiency, we consider a potential lateral removal of the flat, epitaxial absorber structure $(0.8 \mu \mathrm{m}$ for the top cell). In a cathodic corrosion process, where $\mathrm{PH}_{3}$ is formed, three electrons are liberated per $\mathrm{Al} / \mathrm{In}_{\text {atom }}{ }^{26}$, which is equivalent to 12 electrons per crystal unit cell. With a lattice constant $a=0.57 \mathrm{~nm}$, this translates into a charge of $1.04 \mathrm{mC} \mathrm{cm}^{-2}$ for the corrosion of $1 \mathrm{~nm}$. Assuming a (conservative) average current density of $7 \mathrm{~mA} \mathrm{~cm}^{-2}$ during the long-term experiment in Fig. 5 over $40 \mathrm{~h}$ and homogeneous corrosion, this would result in a removal of $1 \mathrm{~mm}$, if all the current would be due to corrosion. As the device still splits water after $40 \mathrm{~h}$, the corroded thickness is certainly $<0.8 \mu \mathrm{m}$, the thickness of the top cell. Hence, the lower boundary of the Faradaic efficiency for $\mathrm{H}_{2}$ evolution can be estimated to be $>99.9 \%$. Gas measurements (Fig. 6a) confirm such a high Faradaic efficiency with the ratio of cathodic to anodic products of 2:1 and the overall amount of gases very close to the expected value derived from the integration of the current.

A general motif of a successful functionalization of InP-based surfaces appears to be the formation of phosphate and phosphite species, simultaneously providing stability as well as efficient charge transfer ${ }^{7,22,23}$. For the AlInP surface studied here, the removal of mixed $\mathrm{Al} / \mathrm{In}$ oxides and the concurrent incorporation of oxygen in the form of $\mathrm{PO}_{x}$ on the first $1-2 \mathrm{~nm}$ is a crucial prerequisite for stability and efficiency. While In-O-In bonds were indeed predicted to form potential charge-carrier recombination centres ${ }^{24}$, the phosphate species seem to provide a similar surface passivation as it was observed in the case of cobalt phosphate on metal-oxide electrodes ${ }^{36}$

For an energetic consideration of the interface formation during functionalization, two junctions have to be regarded, the buried solid-solid interface between the pure, as-grown, AlInP window layer and its oxygen-containing surface layer, as well as the solid-liquid interface between this oxygen-modified surface layer and the electrolyte. In the case of the buried junction, the electron affinities, $\chi$, of the AlInP and the oxide/ $\mathrm{PO}_{x}$ species have to be considered. While AlInP exhibits $\chi=3.8 \mathrm{eV}$ (ref. 18), it changes to $\chi \approx 4.4 \mathrm{eV}$ for indium oxide ${ }^{31}$. Such differences in electron affinity between III-V semiconductors and their oxides with their potential impact on the photovoltage have already been addressed in the literature ${ }^{37}$. The transformation of the surface layer towards a $\mathrm{PO}_{x}$-rich phase with its reduced electron affinity ${ }^{38}$ does, however, shift the conduction band back towards the vacuum level, reducing the band offset at the buried junction. This is supported by XPS, showing a binding energy of the In $3 d$ peak of $443.8,444.2$ and $444.6 \mathrm{eV}$ for the freshly etched, the partly functionalised and the fully functionalised surface, respectively. This trend is confirmed by Mott-Schottky analysis, where the flat band of $-0.7 \mathrm{~V}$ versus RHE for the fully functionalised surface corresponds to $3.9 \mathrm{eV}$ versus the vacuum level, which is very close to what is expected for $\mathrm{n}$-doped AlInP.

Due to the high $\mathrm{n}$-doping in the AlInP and the ultrathin $(<2 \mathrm{~nm})$ oxide/ $\mathrm{PO}_{x}$ layer, the junction between the absorber surface and the $\mathrm{Rh}$ nanoparticles is mainly defined by the Fermi level of the semiconductor ${ }^{39}$, and space charge regions are expected to be thin enough for tunnelling. Consequently, the reduction of the surface recombination velocity by the $\mathrm{PO}_{x}$-rich phase is the main beneficial effect of the surface functionalization.

Our findings suggest that the functionalization should be transferable to other PV-based III-V (tandem) absorbers, which exhibit an InP-containing top layer or an In-rich compound surface. In cases where the absorber does initially not exhibit such a top layer, it is possible to terminate absorber growth with a thin, not necessarily lattice-matched, InP layer on top. As the functionalization mainly depends on the surface chemistry and the Fermi level at the surface (which can be tuned by doping), the 
InP layer could serve as a sacrificial functionalization precursor. Such an approach would consequently widen the material choice for the underlying tandem structure.

With the near-optimum, low light attenuation catalyst loading found here, the amount of Rh for $1 \mathrm{MW}$ electrochemical power output would be in the order of $1 \mathrm{~kg}$. This mass could be considerably reduced by the use of core-shell catalyst nanoparticles prepared, for example, by under potential deposition and galvanic exchange with a core of an earth-abundant material ${ }^{40}$. Further cost reductions from the substrate side could be achieved by the employment of lift-off techniques or the switch to $\mathrm{Si}$ substrates, targeting in combination with light concentration the high-efficiency route to low-cost $\mathrm{H}_{2}$ (ref. 7,9).

The results demonstrate that in situ modification of surfaces enables exceptionally high efficiency for unassisted solar water splitting. The combination of (photo)electrochemical functionalization with surface science analyses provides atomic level insights and feedback for the optimization of the charge transfer processes and interfaces. A further improvement of the functionalization has the potential to achieve even higher stabilities without the need for protective layers by atomic layer deposition, which would introduce additional processing. If the gap between potential (17.4\%) and achieved (14\%) solar-tohydrogen can be further reduced, devices producing solar $\mathrm{H}_{2}$ below $4 \$ / \mathrm{kg}^{9}$ could become feasible.

\section{Methods}

Epitaxial absorber growth and ohmic contact. The tandem absorber was grown epitaxially by metal-organic vapour phase epitaxy on a Ge substrate. The lattice mismatch to the substrate was accounted for by an intermediate step-grading buffer, gradually changing the lattice constant from the substrate to the absorber. For a detailed description, the reader is referred to ref. 19. The ohmic back contact was prepared by evaporating $\mathrm{Ni}$ and $\mathrm{Au}$ on the substrate, followed by annealing.

Etching and chemical oxidation. Before etching, samples were cleaned in 2-propanol and water to remove initial surface contamination. The etching procedure removed the cap layer stopping on the AlInP window layer. The etching solution was composed of $\mathrm{NH}_{4} \mathrm{OH}\left(25 \%\right.$, Sigma Aldrich p.a. grade), $\mathrm{H}_{2} \mathrm{O}_{2}(30 \%$, Merck p.a. grade) and $\mathrm{H}_{2} \mathrm{O}$ in a ratio of $1: 1: 10$ and prepared directly before the etching. All the $\mathrm{H}_{2} \mathrm{O}$ was ultrapure 'Milli-Q' grade. Immediately after the removal of the window layer, samples were rinsed in $\mathrm{H}_{2} \mathrm{O}$, saturated with $\mathrm{O}_{2}$ by purging. The resulting hydrophilic surface was then slowly dried in a stream of $\mathrm{O}_{2}$ for several minutes. Surface transformation was performed in a three-electrode set-up ${ }^{41}$ consisting of a $\mathrm{Pt}$ counter electrode and an $\mathrm{Ag} / \mathrm{AgCl}$ reference electrode in an aqueous $\mathrm{RhCl}_{3}$ solution. Samples were encapsulated in a two-component epoxy resin or, if XPS analysis was performed, clamped against a sealing ring.

Surface transformation and Rh deposition. The electrolyte for functionalization and $\mathrm{Rh}$ deposition was an aqueous solution of $5 \mathrm{mM} \mathrm{Rh}$ (III) chloride trihydrate (99.98\%, Sigma Aldrich) $+0.5 \mathrm{M} \mathrm{KCl} \mathrm{(99.5 \% ,} \mathrm{Alfa} \mathrm{Aesar)}+0.5$ vol\% 2-propanol (Sigma Aldrich p.a. grade). White light was provided by a tungsten iodide lamp. In the three-electrode set-up, the voltage of the working electrode (the sample) is controlled via a potentiostat (PAR Versastat 3) against a reference electrode, in this case $\mathrm{Ag} / \mathrm{AgCl}(+0.197 \mathrm{~V} \text { versus the normal hydrogen electrode })^{41}$. The current then flows between working electrode and a Pt counter electrode immersed in the same solution. Samples were typically embedded into a black two-component epoxy resin. In the cases where XPS analysis was performed, the samples were not embedded into epoxy resin to avoid outgassing and clamped against a sealing O-ring in the polytetrafluoroethylene (PTFE) beaker as the cell compartment.

The surface functionalization was initiated by immersing the sample in the $\mathrm{RhCl}_{3}$ solution under OC conditions in the dark. Illumination with white light caused a shift of the OCP to more positive potentials, modulated by an oscillation. When the OC potential exhibited a maximum, illumination was turned off terminating the photochemical optimization (Fig. 1d). XPS analysis after this step did not reveal any $\mathrm{Rh}(<5 \%$ of a monolayer). Further (photo)electrochemical surface conditioning and catalyst deposition were initiated by the deposition of an $\mathrm{Rh}$ catalyst seed layer in the dark, applying $50 \mathrm{~ms}$ potential pulses at $-0.3 \mathrm{~V}$ versus saturated calomel electrode (SCE) (separated by $1 \mathrm{~s}$ at $-0.1 \mathrm{~V}$ versus SCE) for several minutes (Supplementary Fig. 2). Maintaining the potential pulsing, illumination (also pulsed) was turned on again, leading to an accelerated deposition of $\mathrm{Rh}$ in the form of nanoparticles with an average size of ca. $20 \mathrm{~nm}$ (Fig. 6b). The procedure was completed by another pulsed deposition in the dark.
As the reduction of $\mathrm{Rh}^{3+}$ to metallic $\mathrm{Rh}$ competes with the $\mathrm{H}_{2}$ evolution reaction, the Faradaic efficiency for the deposition is not unity. This explains the transferred charges in the order of $50 \mathrm{mC} \mathrm{cm}^{-1}$, which would otherwise result in a (closed) Rh layer with a thickness in the order of $10 \mathrm{~nm}$. The optimum catalyst loading exhibits a relatively narrow process window: a larger loading results in a higher OC voltage and a steeper onset of the photocurrent, but this increase is over-compensated by a lower photocurrent due to increased light attenuation by the catalyst.

Output characteristic. The set-up for determination of the output characteristics was again a three-electrode set-up with $\mathrm{Pt}$ as counter electrode and $\mathrm{Ag} / \mathrm{AgCl}$ as reference electrode or a two-electrode set-up with a $\mathrm{RuO}_{2}$ counter electrode $\left(1 \mathrm{~cm}^{2}\right)$. A commercial Wacom WXS-50S solar simulator provided the AM 1.5G spectrum at the location of the sample, which was calibrated by quantitatively matching the measured spectrum, which was acquired using an Ocean Optics USB2000 + RAD spectrometer, to AM 1.5G. The PTFE cell (as described in ref. 42) was equipped with a quartz plate as front window and an O-ring of $6 \mathrm{~mm}$ diameter defining the active surface. Electrical connection was assured by a gold finger pressed against the back of the ohmic contact of the solar cell. In the cases, where samples were embedded in epoxy resin, an all-quartz cell was employed. The electrolyte was $1 \mathrm{M} \mathrm{HClO}_{4}$ and, in most cases for the vertical configuration, $1 \mathrm{mM}$ Triton X 100 as a surfactant to reduce the bubble size. Long-term stability in the horizontal set-up, described in the former paragraph, was evaluated without the use of a surfactant. Eighteen hours into the chopped long-term experiment in Fig. 5, an intermediate failure of the chopper, caused by a musca domestica, lead to constant illumination for $2 \mathrm{~h}$.

Statistics: out of 65 analysed samples, 25\% showed current densities beyond $10 \mathrm{~mA} \mathrm{~cm}^{-2}$ and $8 \%$ above $11 \mathrm{~mA} \mathrm{~cm}^{-2}$.

Gas evolution at the electrodes was quantified by a set-up employing two pipettes and manual pressure compensation, after King and $\mathrm{Bard}^{43}$. The cell, containing $1 \mathrm{M} \mathrm{HClO}_{4}$, was first purged with Ar. Afterwards, the solution was saturated with $\mathrm{H}_{2}$ and $\mathrm{O}_{2}$ by dark electrolysis, using the $\mathrm{RuO}_{2}$ counter electrode and an Ir working electrode. Subsequently, the working electrode was switched to the tandem structure, which was then illuminated. The gas volume was measured under ambient conditions.

Photoelectron spectroscopy. For photoelectron spectroscopy, a monochromated $\mathrm{Al} \mathrm{K}_{\alpha}$ source was used. Due to the high photoactivity of the samples inducing a surface photovoltage, energy calibration was achieved by setting the binding energy (relative to the Fermi level $\mathrm{E}_{F}$ ) of the $\mathrm{C} 1 s$ line to $284.8 \mathrm{eV}$. Voigt profiles in combination with a linear background were employed for peak analysis.

For the XPS measurements at different stages of the OCP oscillation (Supplementary Fig. 1), the electrochemical experiment was abruptly interrupted and the sample transferred to Ultra-high vacuum (UHV). To rule out a potential build-up of oxide by subsequent interruptions of the electrochemical experiment, the order of the XPS measurement with respect to maximum/minimum was reversed for different samples, confirming the results.

\section{References}

1. Parkinson, B. \& Turner, J. in Photoelectrochemical Water Splitting: Materials, Processes and Architectures (eds Lewerenz, H.-J. \& Peter, L.) Ch. 1, 1-18 (The Royal Society of Chemistry, 2013).

2. Olah, G. A., Prakash, G. K. S. \& Goeppert, A. Anthropogenic chemical carbon cycle for a sustainable future. J. Am. Chem. Soc. 133, 12881-12898 (2011).

3. Fujishima, A. \& Honda, K. Electrochemical photolysis of water at a semiconductor electrode. Nature 238, 37-38 (1972).

4. Gerischer, H. Heterogeneous electrochemical systems for solar energy conversion. Pure Appl. Chem. 52, 2649-2667 (1980).

5. Bard, A. J. Photoelectrochemistry and heterogeneous photo-catalysis at semiconductors. J. Photochem. 10, 59-75 (1979).

6. Shockley, W. \& Queisser, H. J. Detailed balance limit of efficiency of p-n junction solar cells. J. Appl. Phys. 32, 510-519 (1961).

7. Hannappel, T., May, M. M. \& Lewerenz, H.-J. in Photoelectrochemical Water Splitting: Materials, Processes and Architectures (eds Lewerenz, H.-J. \& Peter, L.) Ch. 9, 223-265 (The Royal Society of Chemistry, 2013).

8. Würfel, P. \& Würfel, U. Physics of Solar Cells: from Basic Principles to Advanced Concepts (John Wiley \& Sons, 2009).

9. Pinaud, B. A. et al. Technical and economic feasibility of centralized facilities for solar hydrogen production via photocatalysis and photoelectrochemistry. Energy Environ. Sci. 6, 1983-2002 (2013).

10. Seger, B. et al. 2-Photon tandem device for water splitting: comparing photocathode first versus photoanode first designs. Energy Environ. Sci. 7, 2397-2413 (2014).

11. Bard, A. J. \& Fox, M. A. Artificial photosynthesis: Solar splitting of water to hydrogen and oxygen. Acc. Chem. Res. 28, 141-145 (1995).

12. Licht, S. et al. Over $18 \%$ solar energy conversion to generation of hydrogen fuel; theory and experiment for efficient solar water splitting. Int. J. Hydrogen Energy 26, 653-659 (2001). 
13. Luo, J. et al. Water photolysis at $12.3 \%$ efficiency via perovskite photovoltaics and earth-abundant catalysts. Science 345, 1593-1596 (2014).

14. Khaselev, O. \& Turner, J. A. A monolithic photovoltaic-photoelectrochemical device for hydrogen production via water splitting. Science 280, 425-427 (1998).

15. Zhai, P. et al. Net primary energy balance of a solar-driven photoelectrochemical water-splitting device. Energy Environ. Sci. 6, 2380-2389 (2013).

16. Ager III, J. W., Shaner, M., Walczak, K., Sharp, I. D. \& Ardo, S. Experimental demonstrations of spontaneous, solar-driven photoelectrochemical water splitting. Energy Environ. Sci. (2015).

17. Hu, S., Xiang, C., Haussener, S., Berger, A. D. \& Lewis, N. S. An analysis of the optimal band gaps of light absorbers in integrated tandem photoelectrochemical water-splitting systems. Energy Environ. Sci. 6, 2984-2993 (2013).

18. Vurgaftman, I., Meyer, J. R. \& Ram-Mohan, L. R. Band parameters for III-V compound semiconductors and their alloys. J. Appl. Phys. 89, 5815-5875 (2001).

19. Dimroth, F., Beckert, R., Meusel, M., Schubert, U. \& Bett, A. W. Metamorphic $\mathrm{Ga}_{y} \mathrm{In}_{1-y} \mathrm{P} / \mathrm{Ga}_{1-x} \mathrm{In}_{x} \mathrm{As}$ tandem solar cells for space and for terrestrial concentrator applications at $\mathrm{C}>1000$ suns. Prog. Photovolt. Res. Appl. 9, 165-178 (2001).

20. Sagol, B. E., Seidel, U., Szabó, N., Schwarzburg, K. \& Hannappel, T. Basic concepts and interfacial aspects of high-efficiency III-V multijunction solar cells. CHIMIA 61, 775-779 (2007).

21. May, M. M. et al. The interface of $\mathrm{GaP}(100)$ and $\mathrm{H}_{2} \mathrm{O}$ studied by photoemission and reflection anisotropy spectroscopy. New J. Phys. 15, 103003 (2013).

22. May, M. M., Lewerenz, H.-J. \& Hannappel, T. Optical in situ Study of $\operatorname{InP}(100)$ Surface Chemistry: Dissociative Adsorption of Water and Oxygen. J. Phys. Chem. C 118, 19032-19041 (2014).

23. Lewerenz, H.-J. et al. Photoelectrocatalysis: principles, nanoemitter applications and routes to bio-inspired systems. Energy Environ. Sci. 3, 748-760 (2010)

24. Wood, B. C., Schwegler, E., Choi, W. I. \& Ogitsu, T. Surface chemistry of $\mathrm{GaP}(001)$ and $\mathrm{InP}(001)$ in contact with water. J. Phys. Chem. C 118, 1062-1070 (2014).

25. Clawson, A. Guide to references on III-V semiconductor chemical etching Mater. Sci. Eng. R-Rep. 31, 1-438 (2001).

26. Khaselev, O. \& Turner, J. A. Electrochemical stability of $\mathrm{p}-\mathrm{GaInP}_{2}$ in aqueous electrolytes toward photoelectrochemical water splitting. J. Electrochem. Soc. 145, 3335-3339 (1998).

27. Aggour, M., Giersig, M. \& Lewerenz, H. Interface condition of n-Si(111) during photocurrent oscillations in $\mathrm{NH}_{4} \mathrm{~F}$ solutions. J. Electroanal. Chem. 383, 67-74 (1995).

28. Grzanna, J., Jungblut, H. \& Lewerenz, H. A model for electrochemical oscillations at the Si-electrolyte contact: Part II. Simulations and experimental results. J. Electroanal. Chem. 486, 190-203 (2000).

29. Möller, K. et al. In-situ monitoring and analysis of $\mathrm{GaSb}(100)$ substrate deoxidation. Appl. Surf. Sci. 242, 392-398 (2005).

30. Chen, G., Visbeck, S. B., Law, D. C. \& Hicks, R. F. Structure-sensitive oxidation of the indium phosphide (001) surface. J. Appl. Phys. 91, 9362-9367 (2002).

31. Muñoz, A. G. et al. Photoelectrochemical conditioning of MOVPE p-InP films for light-induced hydrogen evolution: chemical, electronic and optical properties. ECS J. Solid State Sci. Technol. 2, Q51-Q58 (2013).

32. Li, J., Peat, R. \& Peter, L. Surface recombination at semiconductor electrodes: Part II. Photoinduced "near-surface" recombination centres in $\mathrm{p}-\mathrm{GaP}$. J. Electroanal. Chem. 165, 41-59 (1984).

33. Shaner, M. R., Fountaine, K. T. \& Lewerenz, H.-J. Current-voltage characteristics of coupled photodiode-electrocatalyst devices. Appl. Phys. Lett 103, 143905 (2013).

34. $\mathrm{Hu}$, S. et al. Amorphous $\mathrm{TiO}_{2}$ coatings stabilize Si, GaAs, and $\mathrm{GaP}$ photoanodes for efficient water oxidation. Science 344, 1005-1009 (2014).

35. Kolb, D., Przasnyski, M. \& Gerischer, H. Underpotential deposition of metals and work function differences. J. Electroanal. Chem. 54, 25-38 (1974).
36. Zhong, D. K., Choi, S. \& Gamelin, D. R. Near-complete suppression of surface recombination in solar photoelectrolysis by Co-Pi catalyst-modified $\mathrm{W}: \mathrm{BiVO}_{4}$. J. Am. Chem. Soc. 133, 18370-18377 (2011).

37. Kaiser, B. et al. Solar hydrogen generation with wide-band-gap semiconductors: $\mathrm{GaP}(100)$ photoelectrodes and surface modification. ChemPhysChem 13, 3053-3060 (2012).

38. Robach, Y. et al. Passivation of $\mathrm{InP}$ using $\mathrm{In}\left(\mathrm{PO}_{3}\right)_{3}$-condensed phosphates: from oxide growth properties to metal-insulator-semiconductor field-effecttransistor devices. J. Appl. Phys. 71, 2981-2992 (1992).

39. Calvet, W. et al. Silicon based tandem cells: novel photocathodes for hydrogen production. Phys. Chem. Chem. Phys. 16, 12043-12050 (2014).

40. Zhong, C. J. \& Maye, M. M. Coreshell assembled nanoparticles as catalysts. J. Adv. Mater. 13, 1507-1511 (2001).

41. Bard, A. J. \& Faulkner, L. R. Electrochemical Methods: Fundamentals and Applications 2nd edn (Wiley, 2001).

42. van de Krol, R. \& Grätzel, M. Photoelectrochemical Hydrogen Production Vol. 102 (Springer, 2011)

43. King, D. M. \& Bard, A. J. Coulometric analysis with gas volume measurement. Anal. Chem. 36, 2351-2352 (1964).

\section{Acknowledgements}

We are grateful for support by R. van de Krol and for experimental support by M. Kernbach and H. Kriegel. M. Niemeyer, C. Karcher and J. Ohlmann supported the solar cell development. K. Harbauer and T. Münchenberg assisted with ohmic contact preparation. U. Bloeck performed the TEM measurements, S. Brunken provided the $\mathrm{RuO}_{2}$ counter electrode. We also thank P. Bogdanoff, K. Fountaine and C. McCrory for helpful discussions. M.M.M. acknowledges a scholarship by the Studienstiftung des deutschen Volkes and H.-J.L. acknowledges support by the DFG (project Nr. 1192-3/4) The discussion and interpretation of the data, feedback with experimentation as well as article layout and writing was also supported by the Joint Center for Artificial Photosynthesis, a DOE Energy Innovation Hub, supported through the Office of Science of the US Department of Energy under Award Number DE-SC0004993.

\section{Author contributions}

T.H., H.-J.L. and M.M.M. designed the study. M.M.M. executed the experiments and did the data analysis. D.L. and F.D. prepared the tandem absorber. M.M.M. and H.J.L. wrote the paper and all authors commented on the manuscript.

\section{Additional information}

Supplementary Information accompanies this paper at http://www.nature.com/ naturecommunications

Competing financial interests: The authors declare no competing financial interests.

Reprints and permission information is available online at http://npg.nature.com/ reprintsandpermissions/

How to cite this article: May, M. M. et al. Efficient direct solar-to-hydrogen conversion by in situ interface transformation of a tandem structure. Nat. Commun. 6:8286 doi: $10.1038 /$ ncomms9286 (2015)

This work is licensed under a Creative Commons Attribution 4.0 International License. The images or other third party material in this article are included in the article's Creative Commons license, unless indicated otherwise in the credit line; if the material is not included under the Creative Commons license, users will need to obtain permission from the license holder to reproduce the material To view a copy of this license, visit http://creativecommons.org/licenses/by/4.0/ 